\title{
LSST DETECTOR MODULE AND RAFT ASSEMBLY METROLOGY CONCEPTS*
}

\author{
Peter Z. Takacs ${ }^{\mathrm{a}}$, Paul O'Connor ${ }^{\mathrm{a}}$, Veljko Radeka ${ }^{\mathrm{a}}$, George Mahler ${ }^{\mathrm{b}}$, \\ James Frank ${ }^{c}$, John Geary ${ }^{d}$, and the LSST Camera Team
}

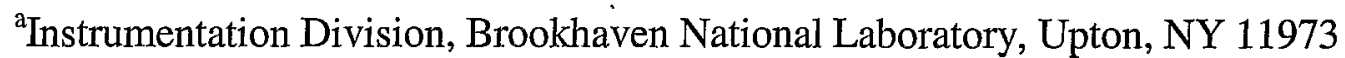

${ }^{b}$ Collider-Accelerator Department, Brookhaven National Laboratory, Upton, NY 11973

${ }^{\mathrm{d}}$ Physics Department, Brookhaven National Laboratory, Upton, NY 11973

May, 2006

"This manuscript has been authored by Brookhaven Science Associates, LLC under Contract No. DE-AC02-98CH10886 with the U.S. Department of Energy. The United States Government retains, and the publisher, by accepting the article for publication, acknowledges, a world-wide license to publish or reproduce the published form of this manuscript, or allow others to do so, for the United States Government purposes. 


\section{DISCLAIMER}

This work was prepared as an account of work sponsored by an agency of the United States Government. Neither the United States Government nor any agency thereof, nor any of their employees, nor any of their contractors, subcontractors, or their employees, makes any warranty, express or implied, or assumes any legal liability or responsibility for the accuracy, completeness, or any third party's use or the results of such use of any information, apparatus, product, or process disclosed, or represents that its use would not infringe privately owned rights. Reference herein to any specific commercial product, process, or service by trade name, trademark, manufacturer, or otherwise, does not necessarily constitute or imply its endorsement, recommendation, or favoring by the United States Government or any agency thereof or its contractors or subcontractors. The views and opinions of authors expressed herein do not necessarily state or reflect those of the United States Government or any agency thereof. 


\title{
LSST Detector Module and Raft Assembly Metrology Concepts
}

\author{
Peter Z. Takacs ${ }^{\mathrm{a}}$, Paul O'Connor ${ }^{\mathrm{a}}$, Veljko Radeka ${ }^{\mathrm{a}}$, George Mahler ${ }^{\mathrm{b}}$, James Frank ${ }^{\mathrm{c}}$, John Geary ${ }^{\mathrm{d}}$, \\ and the LSST camera team \\ ${ }^{\text {a }}$ Instrumentation Division, Brookhaven National Laboratory, PO Box 5000, Upton, NY 11973 \\ ${ }^{\mathrm{b}}$ Collider-Accelerator Dept., Brookhaven National Laboratory, PO Box 5000, Upton, NY 11973 \\ ${ }^{c}$ Physics Department, Brookhaven National Laboratory, PO Box 5000, Upton, NY 11973 \\ ${ }^{\mathrm{d}}$ Harvard Smithsonian CFA, 60 Garden St., Cambridge, MA 02138
}

\begin{abstract}
The LSST camera focal plane array will consist of individual Si sensor modules, each $42 \times 42 \mathrm{~mm}^{2}$ in size, that are assembled into $3 \times 3$ "raft" structures, which are then assembled into the final focal plane array. It is our responsibility at Brookhaven National Lab (BNL) to insure that the individual sensors provided by the manufacturer meet the flatness requirement of $5 \mu \mathrm{m}$ PV and that the assembled raft structure be within the $6.5 \mu \mathrm{m}$ PV flatness tolerance. These tolerances must be measured with the detectors operating in a cryogenic environment at $-100 \mathrm{C}$ in a face-down configuration. Conventional interferometric techniques for flatness testing are inadequate to insure that edge discontinuities between detector elements are within the tolerances because of the quarter-wave phase ambiguity problem. For this reason we have chosen a combination of metrology techniques to solve the discontinuity ambiguity problem that include both a full aperture interferometer and a scanning confocal distance microscope. We will discuss concepts for performing flatness metrology testing with these instruments under these conditions and will present preliminary results of measurement sensitivity and repeatability from tests performed on step height artifacts.
\end{abstract}

Keywords: Metrology, flatness, confocal height microscopy, interferometry, silicon sensor.

\section{INTRODUCTION}

The Large Synoptic Survey Telescope (LSST) presents a formidable challenge to the detector development community. The $8.4 \mathrm{~m}$ diameter telescope is designed to have a fast optical system $(\mathrm{F} / 1.23)$ with a wide field of view $\left(3.5^{\circ}\right)^{1}$. In order to accommodate the large field of view, the current design for the camera focal plane array (FPA), illustrated in Fig. 1, comprises 201 sensor modules, each $42 \times 42 \mathrm{~mm}^{2}$ in area, that are assembled into $3 \times 3$ array "raft" structures, which are then assembled into the final $64 \mathrm{~cm}$ diameter focal plane array (FPA). Each detector module consists of a $4 \mathrm{~K} \mathrm{x}$ $4 \mathrm{~K}$ array of $10 \mu \mathrm{m}$ square pixels, for a total of 3.2 Gpixels in the FPA. Each pixel corresponds to a 0.2 arcsec square

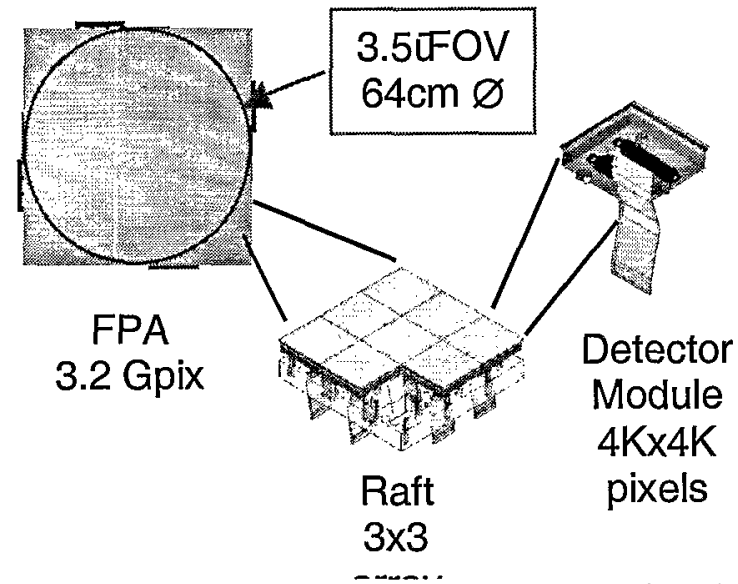

Fig. 1 - The LSST focal plane array is comprised of 21 full rafts assembled from $3 \times 3$ arrays of individual detector modules with $10 \mu \mathrm{m}$ square pixels. region of the sky, which is matched to the expected best seeing conditions at the telescope site. Because of the fast optical system with a low F-number beam, the tolerance on focal plane flatness over the entire FPA is quite stringent: $\Delta \mathrm{z} \leq 10 \mu \mathrm{m}$. The flatness tolerance on each raft assembly is $\Delta z \leq 6.5 \mu \mathrm{m}$ and on each detector module is $\Delta \mathrm{z} \leq 5.0 \mu \mathrm{m}$. Such tight flatness specifications on a focal plane array of this size are at the edge of the current stateof-the-art in flatness metrology $\mathrm{y}^{2-4}$.

$\mathrm{BNL}$ is responsible for overseeing the design and fabrication of the detector modules, assuring that they meet various acceptance criteria such as quantum efficiency, electrical interfaces, and noise performance. Brookhaven is also responsible for assembling the modules into the raft structures. The rafts will be delivered to SLAC at Stanford for assembly into the FPA. It is our responsibility to insure that the assembled raft structures meet the flatness requirements specified above. 


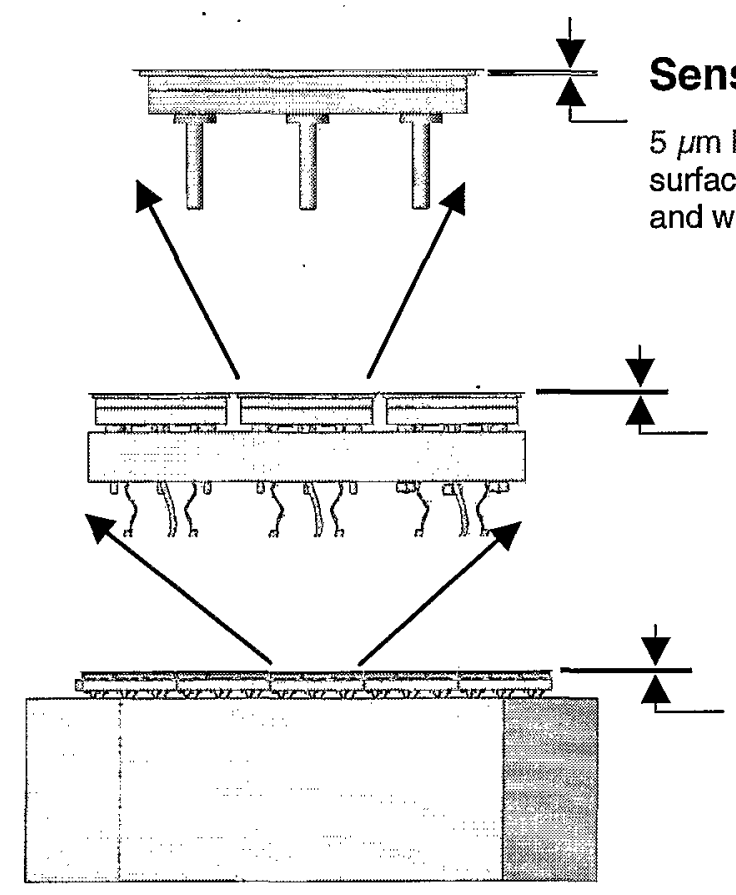

\section{Raft Assembly}

$6.5 \mu \mathrm{m}$ PV over all 9 sensor surfaces (operating, warm or cold, and with $0-45 \mathrm{deg}$ tilt)

\section{Focal Plane Assembly}

$10 \mu \mathrm{m}$ PV over all 201 sensor surfaces (operating, warm or cold, and with 0-45 deg tilt)

Fig. 2 - Flatness tolerances on the various elements that comprise the Focal Plane Assembly (FPA). The actual FPA will be oriented in a face-down configuration. Measurements will need to be made in this attitude and over the range of operating angles.

\section{SENSOR METROLOGY CONSIDERATIONS}

The flatness tolerance requirements on the FPA elements are illustrated schematically in Fig. 2. Sensor modules will be fabricated by one or more commercial vendors according to specifications provided by the Sensor Development Team. A rigorous inspection process is being developed to insure that the delivered modules meet the various optical, mechanical, and electrical design specifications. The two major mechanical specifications that are of concern to BNL are the sensor module flatness and the raft assembly flatness tolerances shown in Fig. 2. Each detector module must be flat to within a $5 \mu \mathrm{m}$ PV (Peak-to-Valley) tolerance and, after assembly into the raft, all 9 modules must be coplanar to within a $6.5 \mu \mathrm{m}$ PV tolerance. These tolerances require a factor of 2 advance over the most recently developed similar sensors ${ }^{2,3}$. Additional assembly tolerance considerations are shown in Fig. 3. Note that the nominal attitude for the FPA is face down when the telescope is pointing at the zenith. We will need to measure the flatness of the raft assemblies in this attitude and at various angles off the zenith. The datum plane for raft assembly is the top surface of the raft structure. The design for this structure is not yet finalized, but it is anticipated that it will be made from a silicon carbide material to provide stiffness with low mass. The surface of this structure can be ground and lapped to a flatness tolerance of on the order of $1 \mu \mathrm{m}$. We can then use this as a datum surface to install each detector module at the same height, $Z_{c}$, as indicated in Fig. 3.

The detector modules will be assembled to the raft structure by means of an adjustable 3-point ball-and-vee kinematic mount system. A concept for this mount system is shown in Fig. 3. This mounting concept was selected for several reasons, Firstly, it relaxes the burden placed on the sensor fabricator to maintain parallelism between the top surface and the datum during the manufacture of the sensor assembly. The primary concern of the fabricator should be to maintain the flatness tolerance during the process of cementing the thin active Si chip to the thick base plate. As long as the sensor surface is flat, any rigid-body tilt can be removed by adjusting the height of the kinematic ball screws. Secondly, the built-in adjustment capability in each ball-end screw cartridge allows one to easily adjust the height of a detector module from the back of the raft without the need to disassemble the mounting pads to install shims or remove material from the mounting interface. The adjustment can be made in real time while monitoring the height of the surface with 


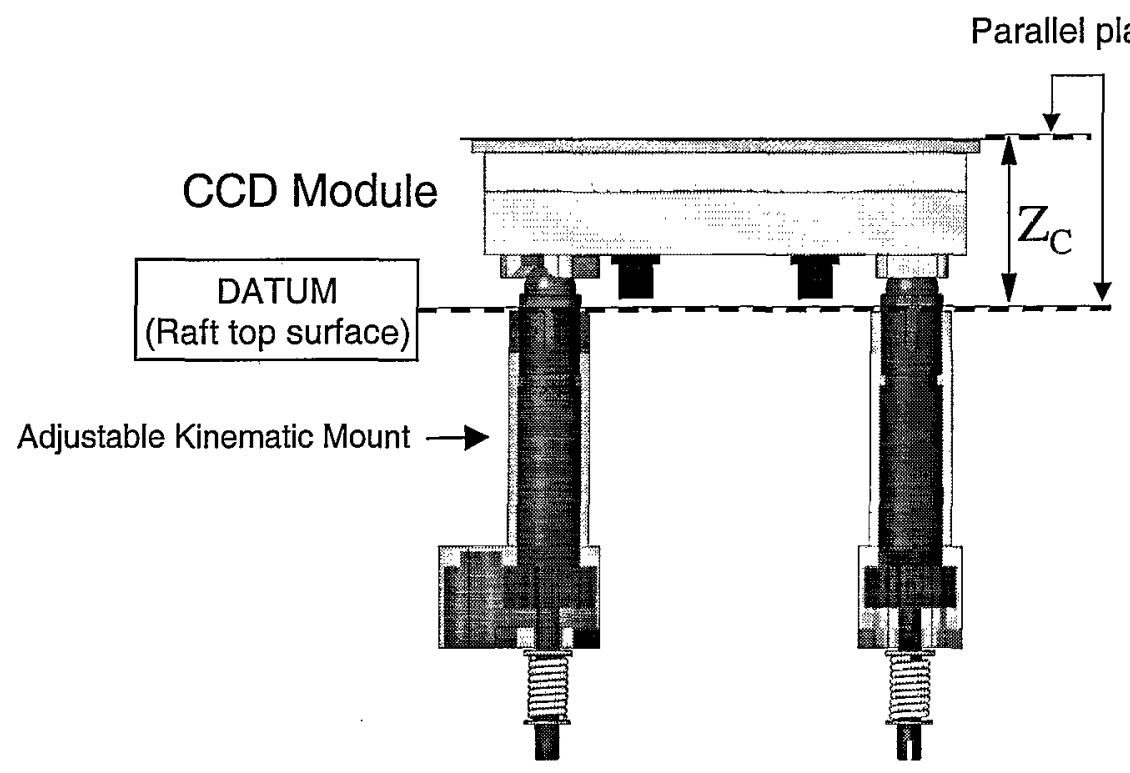

Fig 3 - Adjustable kinematic sensor mounting concept (after Nordby and Guiffre). Each sensor module will be measured relative to a master kinematic jig to determine its departure from planarity. Corresponding ball-end screws can be adjusted to compensate for tilt and height error of the sensor plane.

an appropriate measuring tool. And thirdly, it will allow for easy removal, replacement, and realignment of defective sensors, should the need arise.

The tiled mosaic structure of the rafts and focal plane array complicates the flatness measurement process. The most convenient measurement technique for smooth, flat surfaces is to use phase-measuring interferometry (PMI). However, PMI with conventional long coherence length interferometers will only work on continuous surfaces, i.e. surfaces without gaps or abrupt height discontinuities that are greater than $\lambda / 4$ between adjacent pixels. If such gaps or discontinuities exist, the phase-unwrapping algorithms used by the PMI to convert optical phase to surface height will fail to compute the correct height difference across the gap. Since we are expecting height discontinuities of several microns across the edges of the sensors as they are assembled into the raft, conventional interferometry cannot be used to provide reliable surface height information. A review of the various metrology techniques available for this type of mosaic surface metrology can be found in Ströbele's thesis. ${ }^{5}$ We need to use a non-contact measurement technique that will provide absolute height information. For this reason, we have chosen a relatively newly available Keyence LT$9030 \mathrm{M}$ Confocal Distance Gauge that is mounted onto an X-Y scanning stage to provide a 2D surface profile map over the area of interest. The operating principle of this instrument is based upon a confocal microscope system with a novel internal beam-scanning mechanism to provide measurement along the z-axis. It differs significantly from the triangulation sensor method studied in Ströbele's work. The LT-9030M optical head has a height measurement range of $3 \mathrm{~mm}$ with a standoff distance of $30 \mathrm{~mm}$ from the front of the lens. This standoff distance will allow us to make measurements through the window of the cryostat with the sensor cooled to its operating temperature. of $-100 \mathrm{C}$. The measurement repeatability of the LT-9030M is specified to be $0.1 \mu \mathrm{m}$ under normal conditions. In order to evaluate the suitability of this instrument for use with the LSST sensors, measurements were made on various surfaces to confirm the accuracy and repeatability specifications of the system. These measurements will be discussed below. Once all of the sensor modules have been mounted onto the raft assembly and adjusted for flatness, the Fisba interferometer can be used to monitor the flatness during subsequent handling and thermal cycling tests. The Keyence height measurement provides the absolute calibration across edge discontinuities that may not be resolved correctly by the phase unwrapping algorithm in the Fisba software. We can correct any Fisba errors by means of the Keyence data. 


\section{INSPECTION PROCESS}

Upon arrival at BNL, each sensor module will be inspected for any obvious mechanical defects, such as loose bond wires, or surface or edge damage. Each sensor will then be measured interferometrically to determine its flatness characteristics. A Fisba $200 \mathrm{~mm}$ aperture Twyman-Green phase measuring interferometer is available for this task. The sensors will then be mounted onto a master 3-ball kinematic mount jig that will be fixed onto a master optical flat surface. The absolute surface height of the sensor will be measured relative to the optical flat using the Keyence, with gauge block height standards to transfer the datum level to a height near to the sensor surface, within the $3 \mathrm{~mm}$ measurement range of the Keyence. The Keyence optical head will be mounted on an X-Y translation stage system to perform 2D area scans. Scanning is not required in the Z-direction (vertical) because of the large vertical scan measurement range of the Keyence and the fact that the surfaces should not depart significantly from planar.

Once the mechanical properties of the individual sensor modules have been characterized, they will undergo an extensive series of calibrations and thermal and electronic tests that are not a part of this discussion. During these tests, the sensor modules will be monitored for changes in flatness induced by aging, debonding, or by thermal cycling in the vacuum test cryostat chamber. Flatness monitoring can be done at low temperature through the cryostat window with the Fisba interferometer or with the Keyence system. The Keyence can easily be configured to view the sensor mounted in a face-down configuration, which is the nominal attitude of the FPA when it is in use on the telescope.

Following the individual calibrations, the sensor modules will be assembled into the raft structure. With prior knowledge of the rigid body tilt of each surface, the kinematic mount ball-end screws can be pre-adjusted to minimize the amount of adjustment needed after installation of each module. This assembly will most likely be done with the sensor in a face-down configuration to allow easy access to the adjustment mechanisms and to minimize the potential for surface contamination and accidents.

\section{X-Y SCANNER EVALUATION TESTS}

Height data extracted from the Keyence height-measuring gauge mounted on a scanning X-Y stage requires correction for errors introduced by imperfections in the travel of the translation stages. Mechanical bearing translation stages typically have several microns of height error over travel lengths of hundreds of millimeters. These are specified as straightness and flatness errors in the manufacturer's literature. These are typically caused by long-period bowing and warping errors in the construction of the ways. What is of primary concern to us in the assembly of the raft is the short period error in the mechanical stages caused by imperfections in individual bearings as they engage and disengage from

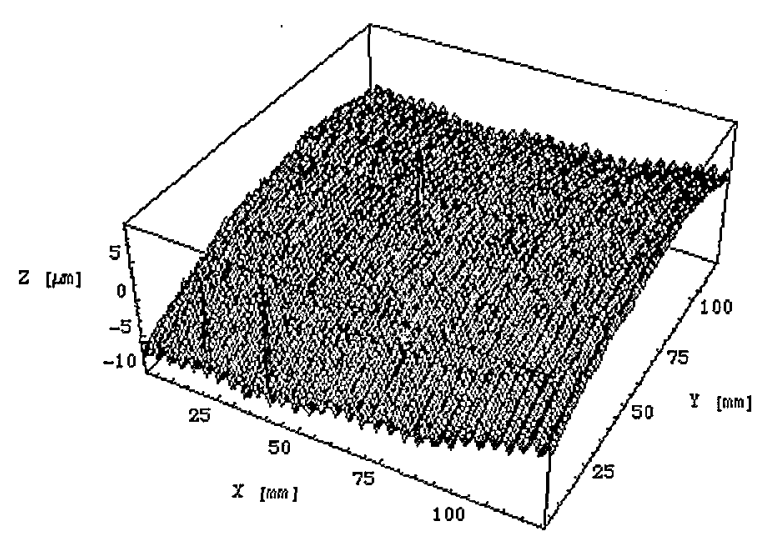

Fig. 4 - Average of 3 scans of the optical flat over $120 \mathrm{~mm} \times 120 \mathrm{~mm}$ area at $1 \mathrm{~mm}$ sampling intervals in each direction.
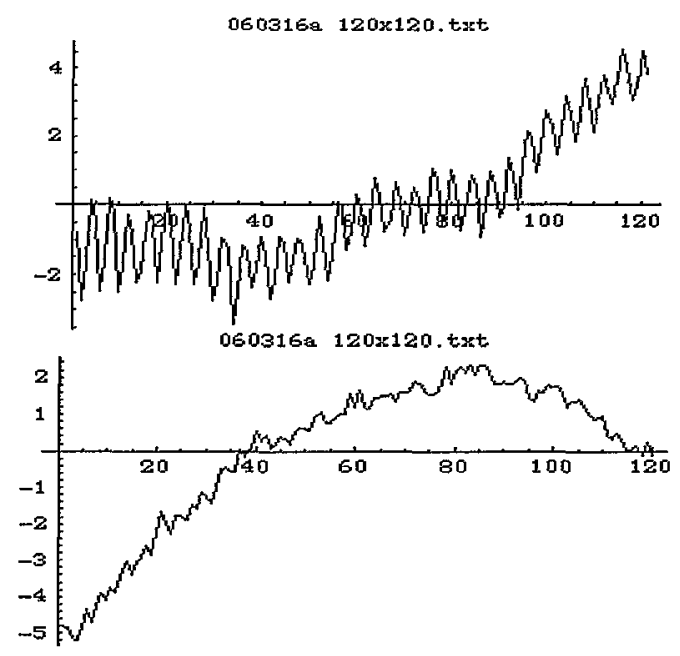

Fig. 5 - Column sums (upper) and row sums (lower) generate mean $x$ - and $y$-profiles from $2 \mathrm{D}$ optical flat data. These show the different error signatures along each axis and provide the LUT correction for each axis. 
the surface of the ways. This chatter error causes short period height fluctuations on the order of several microns over millimeter-length periods. Rapid fluctuations in the height of the stage at these short periods could potentially mask the desired signal that we are trying to measure, namely discontinuities across boundary edges between adjacent sensors. If these errors exist, it is important to know if they are repeatable or if they are random with position in X-Y space. If they are repeatable, one can, in principle, calibrate the error and subtract it from the measurement by means of a look-up table (LUT) correction.

To determine the magnitude and nature of the mechanical bearing-induced errors, we mounted the Keyence optical head onto an Aerotech ATS-3220 open center X-Y translation stage with a $200 \mathrm{~mm} \times 200 \mathrm{~mm}$ scan range. This is a stacked platform of three plates with a $200 \mathrm{~mm}$ square picture frame area cut out in the center of each. The Y-axis stage is sandwiched between the base plate and the $\mathrm{X}$-axis stage on top. Each of the moving platforms is mounted with two sets of recirculating roller bearing tracks and is driven by a stepper motor connected to a precision leadscrew. A $200 \mathrm{~mm}$ diameter optical flat with a surface flatness of better than $\lambda / 20$ over the entire aperture was used as a test object placed in the open space in the center of the stage.

Results from an average of a set of 3 scans are shown in Fig. 4. A definite periodicity is evident along the x-axis direction, but no periodicity is evident along the y-axis direction. This is more apparent after performing a column sum on the averaged input data to produce a mean x-profile (upper frame in Fig. 5) and a row sum to produce a mean yprofile (lower frame). The $x$-axis error exhibits a periodicity of exactly $4 \mathrm{~mm}$, while no periodicity is evident in the $y-$ profile. The repeatability of each scan is quite good. If we subtract the mean at each point from the 3 constituent scans, the standard deviation over all points is $0.33 \mu \mathrm{m}$.

If the errors in the $\mathrm{x}$ and $\mathrm{y}$ axes were independent and are not coupled, we could use the individual column and row sums shown in Fig. 5 as zeroth-order look-up table (LUT) corrections for each point in the scan. This process assumes that the $x$-axis error is independent of position on the $y$-axis, and vice versa, and that the total error is just the sum of the components along the $\mathrm{x}$ and $\mathrm{y}$ axes. This is the simplest possible LUT correction for this scan method. Using the $\mathrm{x}$ and $\mathrm{y}$ profiles generated in Fig. 5, we apply this correction method to another scan with the results shown in Figs. 6 and 7 , with the vertical scales the same as in the corresponding figures 4 and 5. The LUT correction appears to work quite well along the $\mathrm{x}$-axis to reduce the low frequency trend and the periodic error to about $0.4 \mu \mathrm{m}$ RMS from the original level of several microns. The average y-axis profile in Fig. 7 should be a straight line, but it shows a departure from ideal at the low end of the X-axis. Although this simple LUT correction is not perfect, it indicates that a more sophisticated LUT correction method should work to reduce repeatable errors to a low enough level to allow one to use mechanical bearing stages to achieve sub-micron height measurement accuracy with the Keyence optical head.

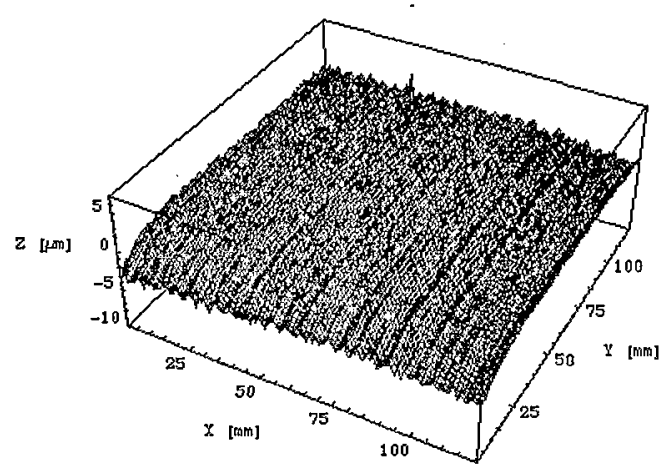

Fig. 6 - Fisba flat scan with LUT correction applied. Departure from planarity is significantly smaller than uncorrected scan in Fig. 4.

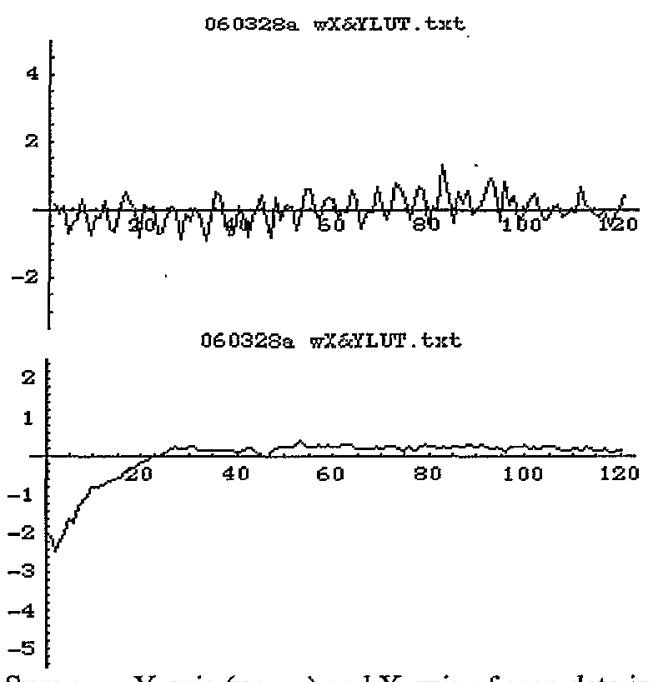

Fig. 7 - Sum over Y-axis (upper) and X-axis of scan data in Fig. 6, showing significant reduction in error with LUT correction. 


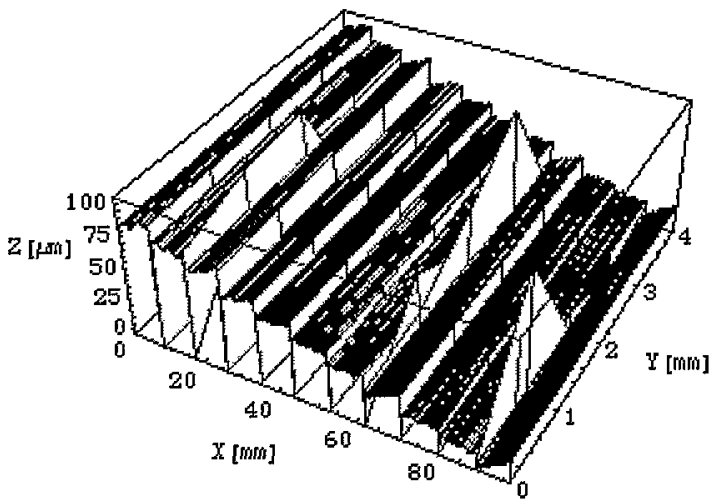

Fig. 8 - Staircase gauge block artifact measurement with LUT correction. Nominal $10 \mu \mathrm{m}$ steps between each gauge block stack.
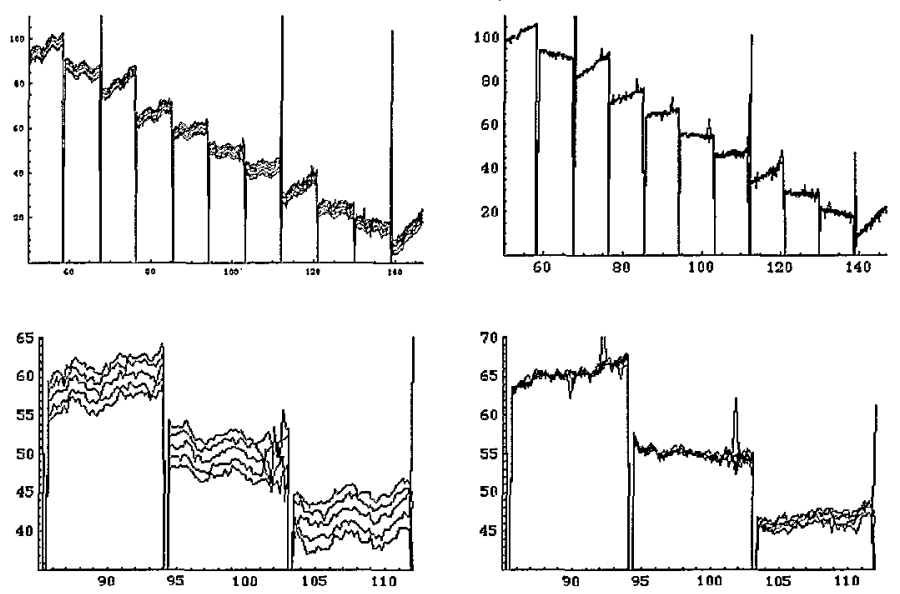

Fig 9 - Staircase artifact scans from 2 runs, without (left column) and with (right column) LUT correction. All 5 scan rows are superimposed here. Scatter in data is significantly reduced with the LUT correction.

\section{STEP HEIGHT DISCONTINUITY MEASUREMENT}

To simulate the expected height discontinuities in the partially-assembled raft, a staircase artifact was assembled from a set of gauge blocks wrung together to produce nominal $10 \mu \mathrm{m}$ steps (except for the lowest step, which is $5 \mu \mathrm{m}$ ). Scans were done on the surface over a region $97 \mathrm{~mm}$ by $5 \mathrm{~mm}$ in area. The step edges were aligned along the $\mathrm{Y}$-axis. The $\Delta X$ step size was $0.2 \mathrm{~mm}$ and the $\Delta \mathrm{Y}$ step size was $1.0 \mathrm{~mm}$. Scans were done with and without LUT correction. The surface map shown in Fig. 8 was done with the LUT correction. Corrections in the $\mathrm{x}$-direction at fractional millimeter positions were generated by cubic spline interpolation from the LUT numbers of the curves in Fig. 5 that are tabulated at integer millimeter positions. The two graphs on the left side of Figure 9 show the large scatter in the data without LUT correction when all 5 scan rows are superimposed. When the LUT correction is used, the slope error producing the offset in the $\mathrm{y}$-direction is eliminated, the ripple in the $\mathrm{x}$-direction is significantly reduced, and all scan row now lies on top of each other with an error of less than one micron. The two sets of scans shown in Fig. 9 are from different scan runs done at different times over the same surface area. It is clear from the scans that the surfaces of the individual gauge blocks are not very flat and are generally wedge-shaped, but the LUT correction allows one to easily see discontinuities across the edges with micron accuracy and precision.

\section{CONCLUSIONS}

The Keyence LT-9030M distance measuring instrument appears to provide sufficient repeatability to allow measurement of height discontinuities at the $0.1 \mu \mathrm{m}$ level. The limiting factor in the measurement is the quality of the $\mathrm{X}$ $\mathrm{Y}$ translation stage. Although there are several microns of high frequency height irregularity in the roller bearings in one of the travel axes of the currently available unit, the deleterious effects on the edge discontinuity measurement can be reduced to below the $1 \mu \mathrm{m}$ level by correction with a look-up table, which is sufficient for our purposes in assembling the raft structures.

\section{ACKNOWLEDGMENTS}

The authors acknowledge the work of Martin Nordby and Gary Guiffre in providing the graphics for the sensor mounting hardware interface. The LSST design and development activity is supported by the National Science Foundation under Scientific Program Order No. 9 (AST-0551161) through Cooperative Agreement AST-0132798. Portions of this work were performed in part under Department of Energy contracts DE-AC02-76SF00515, DE-AC0298CH10886, DE-FG02-91ER40677 and W-7405-Eng-48. Additional funding comes from private donations, in-kind support at Department of Energy laboratories and other LSSTC Institutional Members. 


\section{REFERENCES}

1. R. Allsman et al., "The Large Synoptic Survey Telescope (LSST), A proposal for research and development and preconstruction engineering design", submitted to Dept. of Energy Jan. 20, 2006.

2. Oluseyi, H.M., et al., LBNL 4-Side Buttable CCD Package Development: Jan 2004, in "Sensors and Camera Systems for Scientific, Industrial, and Digital Photography V", Proc. SPIE 5301, eds. M.M. Blouke, N. Sampat, and R.J. Motta, pp. 87-98, (2004).

3. Stover, R.J., et al., Packaging design for Lawrence Berkeley National Laboratory high-resistivity CCDs, presented at Astronomical Telescopes and Instrumentation 2004: Detectors, Structures, Systems Engineering, and Information and Control Technologies, Glasgow, Scotland, in "Optical and Infrared Detectors for Astronomy", Proc. SPIE 5499, eds. J.D. Garnett and J.W. Beletic (2004).

4. Ströbele, S., A new machine for planarity measurement of CCDs and mosaics of CCDs, Experimental Astronomy 11(2), p. 151-156 (2001).

5. Stefan Ströbele, Diplomarbeit, Fachhochschule Ulm (1999).

\section{DISCLAIMER}

This report was prepared as an account of work sponsored by an agency of the United States Government. Neither the United States Government nor any agency thereof, nor any of their employees, nor any of their contractors, subcontractors, or their employees, makes any warranty, express or implied, or assumes any legal liability or responsibility for the accuracy, completeness, or any third party's use or the results of such use of any information, apparatus, product, or process disclosed, or represents that its use would not infringe privately owned rights. Reference herein to any specific commercial product, process, or service by trade name, trademark, manufacturer, or otherwise, does not necessarily constitute or imply its endorsement, recommendation, or favoring by the United States Government or any agency thereof or its contractors or subcontractors. The views and opinions of authors expressed herein do not necessarily state or reflect those of the United States Government or any agency thereof. The publisher by accepting the manuscript for publication acknowledges that the United States Government retains a non-exclusive, paid-up, irrevocable, world-wide license to publish or reproduce the published form of this manuscript, or allow others to do so, for United States Government purposes. 